\title{
Cohomology Vanishing Theorems on Weakly 1-Complete Manifolds
}

\author{
By \\ Takeo OHSAWA*
}

\section{§0. Introduction}

The purpose of the present article is to give an expository account of the works by S. Nakano, A. Kazama, O. Suzuki, and others, on analytic cohomology groups of weakly 1-complete manifolds.

Let $X$ be a paracompact complex manifold of dimension $n$, and let $E$ be a holomorphic vector bundle over $X$. Then, studies on the cohomology groups $H^{q}\left(X, \Omega^{p}(E)\right)$ have significant relationship with function-theoretic and geometric studies of $X$ and $E$. Here $\Omega^{p}(E)$ denotes the sheaf of holomorphic $p$-forms with values in $E$. For example, the following theorem has fundamental importance in the theory of compact complex manifolds.

Theorem K.N. If $X$ is compact and $E$ has a metric whose curvature form is Nakano-positive (cf. Section 2), then

$$
H^{q}\left(X, \Omega^{n}(E)\right)=0, \quad \text { for } \quad q \geqq 1 .
$$

Originally Theorem K.N. was proved for line bundles by K. Kodaira [16], and it was generalized by Nakano [18] for vector bundles of arbitrary rank.

Since Theorem K.N. had so many applications, several mathematicians generalized it to non-compact complex manifolds (cf. Andreotti-Vesentini [4], Grauert-Riemenschneider [11]), and in [20] S. Nakano introduced the concept of weakly 1-complete manifold (cf. Section 1) to establish a vanishing theorem for relatively compact weakly 1-complete domains. Afterwards, A. Kazama [15] generalized Nakano's result and gave a vanishing theorem for weakly 1-complete manifolds, and O. Suzuki [28] gave a different proof in the spirit of Kodaira's origianl work.

Received January 17, 1983.

* Research Institute for Mathematical Sciences, Kyoto University, Kyoto 606, Japan. 
Another important example is Grauert's finiteness theorem on strongly pseudoconvex manifolds. Nakano conjectured that it has a relevant generalization to weakly 1-complete manifolds, which was the motivation of the author's works [23], [24], [26]. They shall be explained in the present article, too.

The author would like to express his sincere thanks to Professors $\mathrm{H}$. Araki and S. Nakano who offered him to write a paper in celebration of the 20-th anniversary of Research Institute for Mathematical Sciences. He also thanks the referee for valuable criticism.

\section{§1. Preliminaries}

\section{Weakly 1-Complete Manifolds}

Let $X$ be a complex manifold of dimension $n . \quad X$ is said to be weakly 1complete if there exists a $C^{\infty}$ function $\varphi: X \rightarrow \mathbf{R}$ which is plurisubharmonic and exhaustive. We shall often say that $(X, \varphi)$ is weakly 1-complete, and set $X_{c}=$ $\{x \in X: \varphi(x)<c\}$.

Proposition 1.1.1. Let $X$ and $Y$ be complex manifolds. Assume that there exists a proper holomorphic map $\pi: X \rightarrow Y$ and that $Y$ is weakly 1-complete. Then $X$ is weakly 1-complete, too.

Proof. Let $\Phi$ be a $C^{\infty}$ plurisubharmonic function on $Y$ which is exhaustive. Then $\pi^{*} \Phi$ is also $C^{\infty}$, plurisubharmonic, and exhaustive.

Proposition 1.1.2. Let $X$ be a strongly pseudoconvex manifold, i.e. a complex manifold provided with an exhaustive function of class $C^{2}$ which is strictly plurisubharmonic outside a compact subset. Then $X$ is weakly 1-complete.

Proof: Let $\psi$ be an exhaustion function of $X$ satisfying the above conditions. Then, regularizing $\psi$ if necessary, we may assume that $\psi$ is of class $C^{\infty}$. Let $c$ be a real number such that $\psi$ is strictly plurisubharmonic on $\{x \in X \mid$ $\psi(x)>c\}$, and let $\lambda: \mathbf{R} \rightarrow \mathbf{R}$ be a $C^{\infty}$ function such that $\lambda(t)=0$ for $t \leqq c$, and $\lambda^{\prime}(t)>0, \lambda^{\prime \prime}(t)>0$ for $t>c$. We put $\varphi(x)=\lambda(\psi(x))$. Then, $\varphi$ is a $C^{\infty}$, plurisubharmonic, and exhaustive function on $X$.

We shall give a relevant generalization of the following theroem in Section 4.

Theorem (Grauert's finiteness theorem. cf. [10]). Let $X$ be a strongly 
pseudoconvex manifold and let $\mathscr{F}$ be a coherent analytic sheaf over $X$. Then, for any $q \geqq 1, H^{q}(X, \mathscr{F})$ is finite dimensional.

Let us recall the basic terminologies in the theory of complex manifolds.

Let $T_{X}$ be the tangent bundle to $X$ and let $T_{X} \otimes \mathrm{C}=T_{X}^{1,0} \oplus T_{X}^{0,1}$ be the splitting into the $\pm \sqrt{-1}$-eigenspaces $T_{X}^{1}, 0, T_{X}^{0,1}$ of the complex structure of $T_{X}$. Let $\sigma$ be a hermitian metric of $X$, i.e., a $C^{\infty}$ section of $\left(T_{X}^{1}, 0\right)^{*} \otimes\left(T_{X}^{0,1}\right)^{*}$ such that $\bar{\sigma}=\sigma$ and $\sigma(v, \bar{v})>0$ for any $v \in T_{X}^{1,0}$ with $v \neq 0$. We shall often regard $\sigma$ as a $C^{\infty}$ section of $\operatorname{Hom}\left(T_{X}^{1,0},\left(T_{X}^{0,1}\right)^{*}\right)$. Let $\omega$ be the image of $\sigma$ under the natural inclusion $\left(T_{X}^{1}, 0\right)^{*} \otimes\left(T_{X}^{0,1}\right)^{*} \hookrightarrow \wedge\left(\Lambda_{X}^{2} \otimes \mathbf{C}\right)$. Then we say that $(X, \sigma)$ is Kählerian if $\omega$ is a $d$-closed form. A hermitian metric provides $X$ with a structure of a metric space. $(X, \sigma)$ is said to be complete if every ball is relatively compact. Here the distance between two points are defined as the infimum of the lengths $\int_{0}^{1} \sqrt{2 \gamma^{*}(\sigma)}$ of differentiable curves $\gamma:[0,1] \rightarrow X$ connecting them.

Proposition 1.1.3. Let $(X, \varphi)$ be a weakly 1-complete manifold with a Kähler metric $\sigma$. Then $X$ has a complete Kähler metric.

Proof. Let $\lambda: \mathbf{R} \rightarrow \mathbf{R}$ be a $C^{\infty}$ convex increasing function such that

$$
\int_{0}^{\infty} \sqrt{\lambda^{\prime \prime}(t)} d t=\infty \text {. }
$$

Then the metric

$$
\begin{aligned}
\sigma_{\lambda}: & =\sigma+\partial \bar{\partial} \lambda(\varphi) \\
& =\sigma+\lambda^{\prime \prime}(\varphi) \partial \varphi \otimes \bar{\partial} \varphi+\lambda^{\prime}(\varphi) \partial \bar{\partial} \varphi
\end{aligned}
$$

is clearly Kählerian. Since $\varphi$ is exhaustive, the completeness follows from (1).

Since every submanifold of $\mathbf{P}^{n}$ admits a Kähler metric, weakly 1-complete submanifolds of $\mathbf{P}^{n}$ admit complete Kähler metrics. In Section 6 we shall take up the problem of projective embeddability of weakly 1-complete manifolds.

\section{Cohomology Groups}

Let $X$ be a paracompact complex manifold of dimension $n$, and let $E \rightarrow X$ be a holomorphic vector bundle of rank $r$. We set $C^{p . q}(X)=\left\{C^{\infty}(p, q)\right.$ forms on $X\}, C^{p \cdot q}(X, E)=\left\{E\right.$-valued $C^{\infty}(p, q)$-forms on $\left.X\right\}, C_{0}^{p, q}(X, E)=$ $\left\{f \in C^{p, q}(X, E) \mid\right.$ support of $f$ is compact $\}$, and $L_{l o c}^{p, q}(X, E)=\{$ locally square integrable $E$-valued $(p, q)$-forms $\}$.

We put $W_{l o c}^{p, q}(X, E)=\left\{f \in L_{l o c}^{p, q}(X, E) \mid f \in L_{l o c}^{p, q+1}(X, E)\right\}$. Then the correspondence 


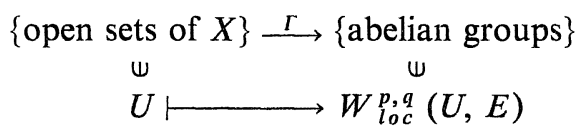

with natural restriction maps $\rho: W_{l o c}^{p, q}(U, E) \rightarrow W_{l o c}^{p, q}(V, E)$ for $V \subset U$ defines a sheaf $\mathscr{W}^{p, q}(E)$ over $X$. Thus we have a complex

$$
0 \longrightarrow \Omega^{p}(E) \longrightarrow \mathscr{W}^{p, 0}(E) \stackrel{\bar{c}}{\longrightarrow} \mathscr{W}^{p, 1}(E) \stackrel{\bar{c}}{\longrightarrow} \cdots \stackrel{\bar{c}}{\longrightarrow} \mathscr{W}^{p, n}(E) \longrightarrow 0,
$$

where $\Omega^{p}(E)$ denotes the sheaf of $E$-valued holomorphic $p$-forms. The proof of the following theorem can be found in [14], but we shall prove it later under a generalized situation.

Theorem 1.2.1. (\#) is an exact sequence of sheaves.

Since $\mathscr{W}^{p, q}(E)$ are fine sheaves (cf. [32]), we have

\section{Corollary 1.2.2.}

$$
\begin{aligned}
& H^{q}\left(X, \Omega^{p}(E)\right) \\
& \quad \cong \Gamma\left(X, \bar{\partial} \mathscr{W}^{p, q-1}(E)\right) / \bar{\partial} W_{l o c}^{p, q-1}(X, E) \\
& \quad=\frac{\left\{f \in L_{l o c}^{p, q}(X, E) \mid \bar{\partial} f=0\right\}}{\left\{f \in L_{l o c}^{p, q}(X, E) \mid \bar{\partial} g=f \text { for some } g \in L_{l o c}^{p, q-1}(X, E)\right\}} .
\end{aligned}
$$

\section{Abstract Vanishing Theorem}

We shall recall here fundamental lemmas due to Hörmander [14].

Let $H_{1}, H_{2} H_{3}$ be three Hilbert spaces with inner products $(,)_{1},(,)_{2}$, $(,)_{3}$, and $T: H_{1} \rightarrow H_{2}, S: H_{2} \rightarrow H_{3}$ be densely defined closed linear operators. We denote by $N_{S}$ the kernel, by $R_{S}$ the range, and by $D_{S}$ the domain of $S$. We shall always assume that $N_{S} \supset R_{T}$. Let $T^{*}, S^{*}$ be the adjoints of $T, S$. Recall that $N_{S} \perp R_{S^{*}}$, hence $R_{S^{4}} \perp R_{T}$. Furthermore,

Lemma 1.3.1. Under the above situation, we have the orthogonal decomposition

$$
H_{2}=\left(N_{S} \cap N_{T^{*}}\right) \oplus \bar{R}_{T} \oplus \bar{R}_{S^{k}} .
$$

Here, $\bar{R}_{T}, \bar{R}_{S^{*}}$ denote the closures of $R_{T}, R_{S^{*}}$ respectively.

Proof. Clearly, $N_{S} \cap N_{T^{*}}, R_{T}, R_{S^{*}}$ are mutually orthogonal. Let $f \perp R_{T}$. Then, for any $u \in D_{T},(T u, f)_{2}=0$. Hence $f \in N_{T^{*}}$. If moreover $f \perp R_{S^{*}}$, then for any $v \in D_{S^{*}}\left(S^{*} v, f\right)=0$. Hence $S f=\left(S^{*}\right)^{*} f=0$, so $f \in N_{T^{*}} \cap N_{S}$.

Theorem 1.3.2 (Abstract vanishing theorem). Let $f \in N_{S}$. Assume that there exists a constant $C$ depending on $f$ such that for any $g \in D_{S} \cap D_{T^{*}}$, 


$$
\left|(f, g)_{2}\right|^{2} \leqq C\left(\left\|T^{*} g\right\|_{1}^{2}+\|S g\|_{3}^{2}\right) .
$$

Then there exists $u$ satisfying $T u=f$ and $\|u\|_{1} \leqq C$. Here \|\|$_{i}$ denote the norms in $H_{i}$.

Proof. In virtue of Hahn-Banach's theorem and Riesz's representation theorem, we have only to prove that

$$
\left|(f, v)_{2}\right|^{2} \leqq C\left\|T^{*} v\right\|_{1}^{2}, \quad \text { for any } \quad v \in D_{T^{*}} .
$$

Let us decompose $v \in D_{T^{*}}$ into the sum $v=v_{1}+v_{2}+v_{3}$, where $v_{1} \in N_{S} \cap N_{T^{*}}$, $v_{2} \in \bar{R}_{T}$ and $v_{3} \in \bar{R}_{S^{4}}$. Since $f \in N_{S},\left(f, v_{3}\right)_{2}=0$. By (3), $\left(f, v_{1}\right)_{2}=0$. Hence $(f, v)_{2}=\left(f, v_{2}\right)_{2}$. Note that $T^{*} v=T^{*} v_{2}$ and that $S v_{2}=0$. Thus we have $\left|(f, v)_{2}\right|^{2} \leqq C\left\|T^{*} v\right\|_{1}^{2}$

Lemma 1.3.3. Assume that from every sequence $\left\{g_{k}\right\}_{k=1}^{\infty} \subset D_{T^{*}} \cap D_{S} \cap$ $\{\|g\|=1\}$ with $\left\|T^{*} g_{k}\right\| \rightarrow 0$ and $\left\|S g_{k}\right\| \rightarrow 0$, one can select a strongly convergent subsequence. Then, $\bar{R}_{T}=R_{T}, \overline{R_{T^{*}}}=R_{T^{*}}$, and $N_{S} \cap N_{T^{*}}$ is a finite dimensional vector space.

Proof. Assume that $R_{T^{*}} \neq \bar{R}_{T^{*}}$. Then there exists a sequence $\left\{u_{k}\right\}_{k=1}^{\infty} \subset D_{T^{*}}$ such that $\left\|u_{k}\right\|=1,\left\|T^{*} u_{k}\right\| \rightarrow 0$ and $u_{k} \perp N_{T^{*}}$. Since $H_{2}=\bar{R}_{T} \oplus N_{T^{*}}$, and $S T=0$, $u_{k} \in N_{S}$. Hence, by assumption $\left\{u_{k}\right\}_{k=1}^{\infty}$ has a subsequence $\left\{u_{k_{v}}\right\}_{v=1}^{\infty}$ which strongly converges to some $u$. Clearly $\|u\|=1, S u=0$ and $u \perp N_{T^{*}}$. Moreover, for any $f \in D_{T}, \quad(T f, u)=\lim \left(T f, u_{k}\right)=\lim \left(f, T^{*} u_{k}\right)=0$. Therefore $T^{*} u=0$, which contradicts the fact that $u \neq 0$ and $u \rightarrow N_{T^{*}}$. Thus we have proved that $R_{T^{*}}=\overline{R_{T^{*}}}$. Next, assume that $R_{T} \neq \overline{R_{T}}$. Then, there exists a sequence $\left\{v_{k}\right\}_{k=1}^{\infty}$ $\subset D_{T}$ such that $\left\|v_{k}\right\|=1,\left\|T v_{k}\right\| \rightarrow 0$, and $v_{k} \perp N_{T}$. Since $R_{T^{*}}=\overline{R_{T^{*}}}$, we can choose a sequence $\left\{w_{k}\right\} \subset D_{T}$ so that $v_{k}=T^{*} w_{h}$ and $\left\|w_{k}\right\| \leqq C$ for some constant $C$. Then we have $0=\lim \left(T v_{h}, w_{k}\right)=\lim \left(T T^{*} w_{k}, w_{k}\right)=\lim \left(T^{*} w_{h}, T^{*} w_{k}\right)$. Hence $\left\|v_{k}\right\|=$ $\left\|T^{*} w_{k}\right\| \rightarrow 0$, which contradicts that $\left\|v_{k}\right\|=1$. Thus $R_{T}=\overline{R_{T}}$. Lastly, by assumption the unit ball in $N_{S} \cap N_{T^{*}}$ is compact, hence $N_{S} \cap N_{T^{*}}$ should be finite dimensional.

\section{Quadratic Forms}

Let $V$ be a real vector space of dimension $2 n$ with a complex structure $J$, and let $V \otimes_{\mathbf{R}} \mathbf{C}=V_{+} \oplus V_{-}$be the decomposition into the eigenspaces $V_{+}, V_{-}$of $J$ for- the eigenvalues $\sqrt{-1},-\sqrt{-1}$, respectively. Let $\sigma \in \operatorname{Hom}\left(V_{+}, \bar{V}_{+}^{*}\right)=$ $V_{+}^{*} \otimes V_{-}^{*}$ be a hermitian metric of $V_{+}$and let $v_{1}, \ldots, v_{n} \in V_{+}^{*}$ be a basis such that $\sigma=\sum_{i=1}^{n} v_{i} \otimes \bar{v}_{i}$. We define the hermitian metrics of $\wedge^{p} V_{+}^{*} \otimes \wedge^{q} V_{-}^{*}$ associated to $\sigma$ 
by the rule that the norms of $v_{I} \otimes \bar{v}_{J}, I=\left(i_{1}, \ldots, i_{p}\right), J=\left(j_{1}, \ldots, j_{q}\right)$ are 1 , where we put $v_{I}=v_{i_{1}} \wedge \cdots \wedge v_{i_{p}}$. We shall often identify $v_{I} \otimes \bar{v}_{J}$ with $v_{I} \wedge \bar{v}_{J}$ via the natural inclusion $\stackrel{p}{\wedge} V_{+}^{*} \otimes \stackrel{q}{\wedge} V_{-}^{*} \hookrightarrow \stackrel{r}{\wedge}\left(V \otimes_{\mathbf{R}} \mathbf{C}\right)$. We put $G=(\sqrt{-1})^{n^{2}} v_{1} \wedge \cdots \wedge$ $v_{n} \wedge \bar{v}_{1} \wedge \cdots \wedge \bar{v}_{n}$. Then $G$ does not depend on the choice of the basis and is left invariant by the complex conjugation. Recalling Laplace's formula for determinants we see that we can define a conjugate linear map $*$ from $\wedge^{p} V_{+}^{*} \otimes \stackrel{q}{\wedge} V^{*}$ to $\stackrel{n-p}{\wedge} V_{+}^{*} \otimes \wedge \wedge \wedge^{n-q}$ by the rule that $\left(v_{I} \wedge \bar{v}_{J}\right) \wedge \bar{*}^{*}\left(v_{I^{\prime}} \wedge \bar{v}_{J^{\prime}}\right)=\operatorname{sgn}\left(\begin{array}{c}I \\ I^{\prime}\end{array}\right) \operatorname{sgn}\left(\begin{array}{l}J \\ J^{\prime}\end{array}\right) G$. Here we put $\operatorname{sgn}\left(\begin{array}{l}i_{1} \cdots i_{p} \\ i_{1}^{\prime} \cdots i_{p}^{\prime}\end{array}\right)=0$ if $\left\{i_{1}, \ldots, i_{p}\right\} \neq\left\{i_{1}^{\prime}, \ldots, i_{p}^{\prime}\right\}$. Note that $* 1=G$. Let $f \in \stackrel{p}{\wedge} V_{+}^{*} \otimes \stackrel{q}{\wedge} V_{-}^{*}$. We denote by $e(f)$ the left multiplication by $f$, and let $L=e(\sqrt{-1} \sigma)$. Let $A$ be the adjoint of $L$. Then we have

Proposition 1.4.1. For any $f \in \stackrel{p}{\wedge} V_{+}^{*} \otimes \wedge^{q} V_{-}^{*}, \bar{*}(\bar{*} f)=(-1)^{p+q} f$ and $\Lambda f=$ $(-1)^{p+q} \bar{*} \bar{*} f$.

Proof. Immediate from the definition.

Let $i(f)$ denote the adjoint of $e(f)$. Then we have $L=\sqrt{-1} \sum_{k=1}^{n} e\left(v_{k}\right) e\left(\bar{v}_{k}\right)$ and $\Lambda=-\sqrt{-1} \sum_{k=1}^{n} i\left(\bar{v}_{k}\right) i\left(v_{k}\right)$. Noting that $i\left(v_{k}\right)\left(v_{k} \wedge v_{I} \wedge \bar{v}_{J}\right)=v_{I} \wedge \bar{v}_{J}$ provided that $k \notin I$, we have

Proposition 1.4.2. For any $f \in \stackrel{p}{\wedge} V_{+}^{*} \otimes \stackrel{q}{\wedge} V_{-}^{*}$,

$$
[L, \Lambda] f=(p+q-n) f \text {, where }[L, \Lambda]=L \Lambda-\Lambda L \text {. }
$$

Proof. An easy computation.

Let $W$ be a complex vector space of dimension $m$ with a hermitian metric $h$, and let $\Theta$ be an element of $\operatorname{Hom}\left(V_{+}, V_{-}^{*}\right) \otimes \operatorname{Hom}(W, W)=V_{+}^{*} \otimes V_{-}^{*} \otimes$ $\operatorname{Hom}(W, W)$. Then the multiplication $e(\Theta)$, as well as $L$ and $\Lambda$, naturally operates on $\sum_{r=0}^{2 n} \Lambda^{r}\left(V \otimes_{\mathbf{R}} \mathbf{C}\right) \otimes W$. We put $\widetilde{\Theta}=\left(\sigma^{-1} \otimes i d_{W}\right) \Theta$. Here we regard $\sigma^{-1} \in \operatorname{Hom}\left(V_{-}^{*}, V_{+}\right)$. Then, $\tilde{\Theta} \in \operatorname{Hom}\left(V_{+} \otimes W, V_{+} \otimes W\right)$. We assume that $\widetilde{\Theta}$ is self-adjoint and positive semi-definite. Let $\gamma$ be the smallest eigenvalue of $\widetilde{\Theta}$. Then we have

Proposition 1.4.2'. For any $f \in\left(\wedge^{n} V_{+}^{*}\right) \otimes V_{-}^{*} \otimes W$,

$$
\langle\sqrt{-1} e(\Theta) \Lambda f, f\rangle \geqq \gamma\langle f, f\rangle .
$$

Here, $\langle$,$\rangle denotes the inner product with respect to \sigma$ and $h$.

Proof. Let $f=\sum_{k=1}^{n}\left(v_{1} \wedge \cdots \wedge v_{n}\right) \wedge \bar{v}_{k} \otimes w_{k}$, where $w_{k} \in W$. Then, $e(\Theta) \wedge f=$ $\sum_{k, l} \Theta_{k l}\left(w_{k}\right) \otimes\left(v_{1} \wedge \cdots \wedge v_{n}\right) \wedge \bar{v}_{l}, \quad$ where $\quad \Theta=\sum_{k, l} \Theta_{k l} v_{k} \wedge \bar{v}_{l}, \quad \Theta_{k l} \in \operatorname{Hom}(W, W)$. Hence, 


$$
\langle\sqrt{-1} e(\Theta) \Lambda f, f\rangle=\sum_{k, l}\left\langle\Theta_{k l}\left(w_{k}\right), w_{l}\right\rangle .
$$

On the other hand we have

$$
\left\langle\widetilde{\Theta}\left(\sum v_{k}^{*} \otimes w_{k}\right), \sum v_{k}^{*} \otimes w_{k}\right\rangle=\sum\left\langle\Theta_{k l}\left(w_{k}\right), w_{l}\right\rangle,
$$

where $v_{1}^{*}, \ldots, v_{n}^{*}$ denotes the dual basis to $v_{1}, \ldots, v_{n}$. Therefore,

$$
\begin{aligned}
& \langle\sqrt{-1} e(\Theta) \Lambda f, f\rangle \\
& \quad \geqq \gamma\left\langle\sum v_{k}^{*} \otimes w_{k}, \sum v_{k}^{*} \otimes w_{k}\right\rangle \\
& \quad=\gamma\langle f, f\rangle .
\end{aligned}
$$

Generalizing the above proposition we have

Proposition 1.4.3. Let $\gamma_{q}$ be the supremum of

$$
\inf _{u \in S \otimes W, u \neq 0}\langle\widetilde{\Theta}(u), u\rangle \mid\langle u, u\rangle,
$$

where $S$ runs over $(q-1)$-codimensional linear subspaces of $V_{+}$. Then, $\langle\sqrt{-1} e(\Theta) \Lambda f, f\rangle \geqq \gamma_{q}\langle f, f\rangle$, for any $f \in\left(\stackrel{n}{\wedge} V_{+}^{*}\right) \otimes\left(\stackrel{q}{\wedge} V_{-}^{*}\right)$.

Proof. Similar as above. For the detail the reader is referred to [26].

Let $\sigma^{\prime} \in \operatorname{Hom}\left(V_{+}, V_{-}^{*}\right)=V_{+}^{*} \otimes V_{-}^{*}$. Assume that $\bar{\sigma}^{\prime}=\sigma^{\prime}$ and $\sigma^{\prime}(v, \bar{v}) \geqq 0$ for any $v \in V_{+}$. Let $\gamma^{\prime}$ be the smallest eigenvalue of $\tilde{\Theta}^{\prime}:=\left(\left(\sigma+\sigma^{\prime}\right)^{-1} \otimes i d_{W}\right)$ 。 $\left(\Theta+\sigma^{\prime} \otimes i d_{W}\right)$.

Proposition 1.4.4. Under the above situation, we have

$$
\gamma^{\prime} \geqq \min (\gamma, 1) \text {. }
$$

Proof. Given any $\sigma^{\prime}$ as above, we can choose $v_{1}, \ldots, v_{n} \in V_{+}^{*}$ so that $\sigma=$ $\sum v_{1} \otimes \bar{v}_{i}$ and $\sigma^{\prime}=\sum \lambda_{i} v_{i} \otimes \bar{v}_{i}, \lambda_{i} \geqq 0 . \quad$ By (5), we have

$$
\begin{aligned}
& \left\langle\tilde{\Theta}^{\prime}\left(\sum v_{k}^{*} \otimes w_{k}\right), \sum v_{k}^{*} \otimes w_{k}\right\rangle \\
& \quad=\sum_{k, l}\left\langle\Theta_{k l}\left(\sqrt{\frac{1}{1+\lambda_{k}}} w_{k}\right), \sqrt{\frac{1}{1+\lambda_{l}}} w_{l}\right\rangle+\sum \frac{\lambda_{k}}{1+\lambda_{k}}\left\langle w_{k}, w_{k}\right\rangle,
\end{aligned}
$$

where the inner product in the left hand side is with respect to $\sigma+\sigma^{\prime}$. Noting that

$$
\begin{aligned}
& \sum_{k, l}\left\langle\Theta_{k l}\left(\sqrt{\frac{1}{1+\lambda_{k}}} w_{k}\right), \sqrt{\frac{1}{1+\lambda_{l}}} w_{l}\right\rangle \\
& \geqq \gamma \sum \frac{1}{1+\lambda_{k}}\left\|w_{k}\right\|^{2},
\end{aligned}
$$

we have 


$$
\begin{aligned}
& \left\langle\widetilde{\Theta}^{\prime}\left(\sum v_{k}^{*} \otimes w_{k}\right), \sum v_{k}^{*} \otimes w_{k}\right\rangle \\
& \quad \geqq \sum \frac{\gamma+\lambda_{k}}{1+\lambda_{k}}\left\|w_{k}\right\|^{2} \\
& \geqq \min (1, \gamma) \sum\left\|w_{k}\right\|^{2} .
\end{aligned}
$$

Clearly the above propositions are applicable to hermitian vector bundles. In the following sections we apply the above propositions for $T_{X}$ and $E$ in place of $V$ and $W$.

\section{§2. A Priori Estimates on Complete Kähler Manifolds}

\section{Approximation Principle of Andreotti-Vesentini}

Let $(X, \sigma)$ be a hermitian manifold, let $(E, h)$ be a hermitian vector bundle over $X$, and let $\left\{e_{i j}\right\}$ be a system of transition functions of $E$ associated to a trivializing covering $\left\{U_{i}\right\}$. Then $h$ is represented by a system $\left\{h_{i}\right\}$ of hermitian matrix-valued $C^{\infty}$ functions satisfying $h_{i}={ }^{t} \bar{e}_{j i} h_{j} e_{j i}$ on $U_{i} \cap U_{j}$. Let $d v$ be the volume form with respect to the Riemannian metric $2 \operatorname{Re} \sigma$ on the underlying differentiable manifold $X$. Then, $d v=\bar{*} 1$ and $|f|^{2} d v={ }^{t} f_{i} \wedge{ }_{*} h_{i} f_{i}$, where $f=$ $\left\{f_{i}\right\} \in C^{p, q}(X, E)$ and $f_{i}$ are vectors of $(p, q)$-forms on $U_{i}$ satisfying $f_{i}=e_{i j} f_{j}$ on $U_{i} \cap U_{j}$. Therefore the (formal) adjoint $\vartheta_{h}$ of $\bar{\partial}$ is given by

$$
\vartheta_{h} f=-\bar{*}^{\prime} h_{i}^{-1} \bar{c} \bar{h}_{i} \bar{*} f .
$$

We define a norm \|\| in $C_{0}^{p, q}(X, E)$ by $\|f\|^{2}=\int_{X}|f|^{2} d v$. Let $x_{0}$ be a point of $X$ and let $\rho(x)=\operatorname{dist}\left(x_{0}, x\right)$, the distance between $x_{0}$ and $x$. Then, by the triangle inequality $\rho$ is a Lipschitz continuous function with Lipschitz constant 1 . Let $L^{p, q}(X, E)$ be the completion of $C_{0}^{p, q}(X, E)$ with respect to \|\| , and let $\bar{o}$ : $L^{p . q}(X, E) \rightarrow L^{p \cdot q+1}(X, E)$ be the extension of $\overline{\hat{c}}$ with domain $D_{\overline{\bar{c}}}^{p, q}=$ $\left\{f \in L^{p, q}(X, E) \mid \bar{\partial} f \in L^{p, q+1}(X, E)\right\}$. Here $\bar{c} f \in L^{p, q+1}(X, E)$ should read "there exists $u \in L^{p, q+1}(X, E)$ such that $(u, \varphi)=\left(f, \vartheta_{h} \varphi\right)$ for any $\varphi \in C_{0}^{p, q+1}(X, E)$ "'. Then, recalling the usual regularization method we see that, for any $f \in D_{\vec{\partial}}^{p, q}$ one can find a sequence $\{\varphi\}_{k=1}^{\infty} \subset C_{0}^{p, q}(X, E)$ such that on any compact subset $K \subset X$, $\varphi_{k}$ and $\bar{\partial} \varphi_{k}$ strongly converge to $f$ and $\bar{\partial} f$, respectively. Thus, regularizing $\left\{\rho(x / r) \varphi_{k_{r}}\right\}_{r=1}^{\infty}\left(k_{1} \ll k_{2} \ll \cdots\right)$ again, we obtain the following

Proposition 2.1.1. If $(X, \sigma)$ is a complete hermitian manifold, then $C_{0}^{p, q}(X, E)$ is dense in $D_{\bar{\partial}}^{p, q}$ with respect to the norm $\|u\|+\|\bar{\partial} u\|$. 
Let $\bar{\partial}^{*}$ be the adjoint of $\bar{c}: L^{p, q}(X, E) \rightarrow L^{p, q+1}(X, E)$. Then, similarly we have

Proposition 2.1.2. If $(X, \sigma)$ is a complete hermitian manifold, then $C_{0}^{p, q}(X, E)$ is dense in $D_{\bar{\partial}^{*}}^{p, q}$ with respect to the norm $\|u\|+\left\|\bar{\partial}^{*} u\right\|$. Moreover, $C_{0}^{p, q}(X, E)$ is dense in $D_{\overline{\hat{\theta}}}^{p, q} \cap D_{\overline{\tilde{c}}^{*}}^{p, q}$ with respect to the norm $\|u\|+\|\bar{\partial} u\|+\left\|\bar{\partial}^{*} u\right\|$.

For the detail of the proof, the reader is referred to [5]. We shall call Proposition 2.1.1 and Proposition 2.1.2 the approximation principle.

When we need to indicate $\sigma$ and $h$, we denote $\|f\|_{h, \sigma}, L^{p, q}(X, E, h, \sigma)$, etc.

\section{A Priori Estimates}

Let the notations be as above. We set $\Theta_{i}=-\bar{\partial}\left(h_{i}^{-1} \partial h_{i}\right)$. Then $\left\{\Theta_{i}\right\}$ defines an element $\Theta_{h}$ of $C^{1,1}(X, \operatorname{Hom}(E, E)) . \Theta_{h}$ is called the curvature form of $h$.

Proposition 2.2.1. Let $(X, \sigma)$ be a Kähler manifold and let $(E, h)$ be a hermitian vector bundle over $X$. Then we have

$$
\begin{aligned}
& \|\bar{\partial} f\|^{2}+\left\|\vartheta_{h} f\right\|^{2} \\
& \quad \geqq\left(\sqrt{-1}\left[e\left(\Theta_{h}\right), \Lambda\right] f, f\right),
\end{aligned}
$$

for any $f \in C_{0}^{p, q}(X, E)$.

Proof. We put $\bar{\vartheta}:=-\bar{*} \partial \bar{*}: C^{p, q}(X, E) \rightarrow C^{p-1, q}(X, E)$. Let $\partial_{h}$ be the adjoint of $\bar{\partial}$ with respect to $\sigma$ and $h$. Then we have $\left(\partial_{h} f\right)_{i}=h_{i}^{-1} \partial\left(h_{i} f_{i}\right),[\Lambda, \bar{\partial}]=$ $\sqrt{-1} \bar{\vartheta}$, and $\left[\Lambda, \partial_{h}\right]=-\sqrt{-1} \vartheta_{h}$. Hence we have

$$
\begin{aligned}
\bar{\partial} \vartheta_{h} & +\vartheta_{h} \bar{\partial} \\
= & \bar{\partial}\left(\sqrt{-1}\left[\Lambda, \partial_{h}\right]\right)+\left(\sqrt{-1}\left[\Lambda, \partial_{h}\right]\right) \bar{\partial} \\
= & \sqrt{-1}[\bar{\partial}, \Lambda] \partial_{h}+\sqrt{-1} \Lambda \bar{\partial} \partial_{h}-\sqrt{-1} \bar{\partial} \partial_{h} \Lambda \\
& +\sqrt{-1} \partial_{h}[\bar{c}, \Lambda]-\sqrt{-1} \partial_{h} \bar{\partial} \Lambda+\sqrt{-1} \Lambda \partial_{h} \bar{\partial} \\
= & \vartheta \partial_{h}+\partial_{h} \vartheta+\left[-\sqrt{-1}\left(\bar{\partial} \partial_{h}+\partial_{h} \bar{\partial}\right), \Lambda\right],
\end{aligned}
$$

and

$$
\begin{aligned}
- & \sqrt{-1}\left(\bar{\partial} \partial_{h}+\partial_{h} \bar{\partial}\right) f \\
& =\sqrt{-1}\left(-\bar{\partial} h_{i}^{-1} \partial\left(h_{i} f_{i}\right)-h_{i}^{-1} \partial\left(h_{i} \bar{\partial} f_{i}\right)\right) \\
& =\sqrt{-1}\left(-\bar{\partial} \partial f_{i}-\bar{\partial}\left(h_{i}^{-1} \partial h_{i} f_{i}\right)-\partial \bar{\partial} f_{i}-\left(h_{i}^{-1} \partial h_{i}\right) \bar{\partial} f_{i}\right) \\
& =e\left(\Theta_{h}\right) f .
\end{aligned}
$$

Thus we obtain 


$$
\begin{aligned}
& (\bar{\partial} f, \bar{\partial} f)+\left(\vartheta_{h} f, \vartheta_{h} f\right) \\
& \quad=(\bar{\vartheta} f, \bar{\vartheta} f)+\left(\partial_{h} f, \partial_{h} f\right)+\left(\sqrt{-1}\left[e\left(\Theta_{h}\right), \Lambda\right] f, f\right) \\
& \quad \geqq\left(\sqrt{-1}\left[e\left(\Theta_{h}\right), \Lambda\right] f, f\right),
\end{aligned}
$$

for $f \in C_{0}^{p, q}(X, E)$.

By the approximation principle we have

Proposition 2.2.2. If $(X, \varphi)$ is complete and Kählerian, then for any hermitian bundle $(E, h)$ over $X$,

$$
\begin{aligned}
& \|\bar{\partial} f\|^{2}+\left\|\bar{\partial}^{*} f\right\|^{2} \\
& \quad \geqq\left(\sqrt{-1}\left[e\left(\Theta_{h}\right), \Lambda\right] f, f\right), \quad \text { for } \quad f \in D_{\partial}^{p, q} \cap D_{\bar{\partial}^{*}}^{p, q} .
\end{aligned}
$$

Combining Proposition 2.2.2 with Abstract vanishing theorem (Theorem 1.3.2), we obtain

Theorem 2.2.3. Let $(X, \sigma)$ be a complete Kähler manifold, and let $(E, h)$ be a hermitian vector bundle over $X$. Assume that for some $(p, q)$ we have

$$
\begin{aligned}
\left(\sqrt{-1}\left[e\left(\Theta_{h}\right), \Lambda\right] f, f\right) \geqq & (c(x) f, f), \\
& \text { for any } f \in C_{0}^{p, q}(X, E),
\end{aligned}
$$

where $c(x)$ is a positive continuous function on $X$. Then, for any $g \in L^{p, q}(X, E)$ satisfying $\bar{\partial} g=0$ and $\int_{X} c(x)^{-1}|g|^{2} d v<\infty$, we can find $u \in L^{p, q-1}(X, E)$ such that $\bar{\partial} u=g$ and $\|u\|^{2} \leqq \int_{X} c(x)^{-1}|g|^{2} d v$.

Let the smallest eigenvalue of $\left(\sigma^{-1} \otimes i d_{E}\right) \Theta_{h}$ at $x \in X$ be $\gamma_{h}(x)$. Clearly $\left(\sigma^{-1} \otimes i d_{E}\right) \Theta_{h}$ is self-adjoint. Then $\gamma_{h}$ is a continuous function on $X .(E, h)$ is said to be Nakano-positive if $\gamma_{h}>0$ everywhere. There is another notion of positivity due to Griffiths [12]. They agree when $r=1$ and coincides with the classical notion of positivity due to Kodaira [16], so we say simply 'positive' for line bundles. Note that Nakano-positivity does not depend on the choice of $\sigma$, so that we can say " $(E, h)$ is Nakano-positive". We say $\Theta_{h}$ is Nakanopositive at $x$ if $\gamma_{h}(x)>0$.

Theorem 2.2.4. If $(E, h)$ is a Nakano-positive bundle over a complete Kähler manifold $(X, \sigma)$, then for any $g \in L^{n, q}(X, E), q \geqq 1$, satisfying $\bar{\partial} g=0$ and $\int_{X} \gamma_{h}^{-1}|g|^{2} d v<\infty$, we can find $u \in L^{n, q-1}(X, E)$ such that $\bar{\partial} u=g$ and $\|u\|^{2} \leqq$ $\int_{X} \gamma_{h}^{-1}|g|^{2} d v$

Proof. Immediate from Proposition 1.4.3. 


\section{§3. Vanishing Theorems on Weakly 1-Complete Manifolds}

Let $(X, \varphi)$ be a weakly 1-complete manifold of dimension $n$ with a Kähler metric $\sigma$, and $(E, h)$ a hermitian bundle over $X$. Let the notations $\gamma_{h}$, $\Theta_{h}$, etc. be as in Section 2.

Lemma 3.1. For any $C^{\infty}$ convex increasing function $\lambda, \gamma_{h} \leqq \gamma_{h \exp (-\lambda(\varphi))}$.

Proof. Immediate from the definition.

Lemma 3.2. For any positive continuous function $\mu: X \rightarrow \mathbf{R}$, we can find a $C^{\infty}$ convex increasing function $\lambda: \mathbf{R} \rightarrow \mathbf{R}$ satisfying $\int_{X} e^{-\lambda(\varphi)} \mu d v<\infty$.

Proof. Trivial.

From these two Lemmas we obtain

Proposition 3.3. Assume that $(E, h)$ is Nakano-positive. Then, for any $g \in L_{\text {loc }}^{p, q}(X, E)$, there exists a convex increasing $C^{\infty}$ function $\lambda: \mathbf{R} \rightarrow \mathbf{R}$ such that $\int_{X} \gamma_{h \exp (-\lambda(\varphi))}{ }^{-1} e^{-\lambda(\varphi)}|g|^{2} d v<\infty$.

Since $e^{-\lambda(\varphi) / 2}|g|$ is the length of $g$ with respect to $\sigma$ and $h e^{-\lambda(\varphi)}$, Theorem 2.2.4 implies now immediately the following

Theorem 3.4. Let $X$ be a weakly 1-complete Kähler manifold of dimension $n$, and let $(E, h)$ be a Nakano-positive bundle over $X$. Then, for any $g \in L_{l o c}^{n, q}(X, E), q \geqq 1$, satisfying $\bar{\partial} g=0$, there exist $u \in L_{l o c}^{n, q-1}(X, E)$ such that $\bar{\partial} u=g$.

Remark 3.5. If $(E, h)$ is Nakano-positive, then the line bundle (det $E$, $\operatorname{det} h$ ) is also positive, so that $\Theta_{\operatorname{det} h}$ defines a Kähler metric on $X$. Thus Kähler-condition is implicit in the positivity assumption of $(E, h)$.

The ball $\mathbf{B}^{n}=\left\{z \in \mathbf{C}^{n} \mid\|z\|<1\right\}$ is weakly 1-complete with respect to $\varphi=$ $-\log \left(1-\|z\|^{2}\right)$. Moreover the trivial bundle over $\mathbf{B}^{n}$ is clearly positive. Thus we have proved Theorem 1.2.1, and hence Theorem 3.4 implies the following theorem which is first due to Kazama [15] (cf. also Nakano [20] and Suzuki [28]).

Theorem 3.6. Let $X$ be a weakly 1-complete manifold of dimension $n$, and let $(E, h)$ be a Nakano-positive bundle. Then,

$$
H^{q}\left(X, \Omega^{n}(E)\right)=0, \quad \text { for } \quad q \geqq 1 .
$$


For positive line bundles we can say more.

Theorem 3.7 (Nakano [21]). Let $(X, \varphi)$ be a weakly 1-complete manifold of dimension $n$, and let $(B, a)$ be a positive line bundle over $X$. Then,

$$
H^{q}\left(X, \Omega^{p}(B)\right)=0 \text {, when } p+q>n .
$$

Proof. First we prepare sublemmas.

Sublemma 1. Let $\mu(t)$ be a continuous function on $\mathbf{R}$. Then there exists an entire analytic function $f: \mathbf{C} \rightarrow \mathbf{C}$ such that $f$ is real valued on $\mathbf{R}$ and $f(t)>\mu(t)$.

Proof. Choose a sequence $\left\{\mu_{k}\right\}_{k=0}^{\infty}$ of integers such that $\mu_{k}>k$ and $t^{\mu_{k}}>$ $2^{(k-1) \mu_{k}} \mu(t)$, for $2^{k} \leqq t \leqq 2^{k+1}$. Then the power series $\sum_{k=0}^{\infty} 2^{(1-k) \mu_{k}} z^{\mu_{k}}+\sup _{-1 \leqq t \leqq 1} \mu(t)$ defines an entire function $f$ satisfying the requirement.

Sublemma 2. Let $\left\{c_{k}\right\}_{k=0}^{\infty}$ be a sequence of positive real numbers. Assume that there exists an integer $m$ such that $\left\{c_{k}\right\}_{k \geqq m}$ is monotonically decreasing and that $\lim c_{k}^{1 / k}=0$. Then, $n c_{n} \leqq \sum_{k=0}^{n-1} c_{k} c_{n-k-1}$, for $n \gg 0$.

Proof. Easy.

Note that for any entire function $f$ we have

$$
|f(t)| \leqq \sum_{k \geqq 0} \frac{\left|f^{(k)}(0)\right|}{k !} t^{k} \leqq \sum_{k \geqq 0} c_{k} t^{k} \quad \text { for } \quad \mathrm{t}>0 .
$$

Here we set

$$
c_{k}=\sup _{m \geqq k}\left(m \sqrt{\frac{f^{(m)}(0)}{m !}}\right)^{k}
$$

Thus, combining these two sublemmas we obtain

Sublemma 3. For any continuous function $\mu(t)$ on $\mathbf{R}$, we can find a convex increasing $C^{\infty}$ function $f$ on $\mathbf{R}$ such that $f(t)>\mu(t)$ for $t>0,(f(t))^{2}>f^{\prime}(t)$ on $(K, \infty)$, and $(f(t))^{4}>f^{\prime \prime}(t)$ on $(K, \infty)$, where $K$ is a positive number depending on $\mu(t)$.

Returning to the proof of Theorem 3.7, let $f \in L_{l o c}^{p, q}(X, B), p+q>n$, and $\bar{\partial} f=0$. We put $\tilde{a}=a \exp \left(-\varphi^{2}\right)$. Then $\Theta_{\tilde{a}}=\Theta_{a}+2(\partial \varphi \otimes \bar{\partial} \varphi+\partial \bar{\partial} \varphi)$ gives a complete Kaehler metric $\tilde{\sigma}$ on $X$. Let $d \tilde{v}$ be the associated volume form, and fix a continuous function $\rho(t)$ on $\mathbf{R}$ such that $\int_{X} e^{-\rho(\varphi)}|f|_{\tilde{a}, \tilde{\sigma}}^{2} d \tilde{v}<\infty$. By Sublemma 3, we can find a constant $K$ and $a C^{\infty}$ convex increasing function $\lambda$ : $\mathbf{R} \rightarrow \mathbf{R}$ such that $\lambda(t)>2 \rho(t)$ for $t>0,(\lambda(t))^{2}>\lambda^{\prime}(t)$ on $(K, \infty)$, and $(\lambda(t))^{4}>\lambda^{\prime \prime}(t)$ 
on $(K, \infty)$. We put $\sigma_{\lambda}=\tilde{\sigma}+\partial \bar{\partial} \lambda(\varphi), a_{\lambda}=\tilde{a} \exp (-\lambda(\varphi))$, and $d v_{\lambda}=$ the volume form with respect to $\sigma_{\lambda}$. Then we have

$$
d v_{\lambda}=\prod_{i=1}^{n}\left(1+\lambda_{i}\right) d \tilde{v} .
$$

Here $\lambda_{i}$ denote the eigenvalues of $\partial \bar{\partial} \lambda(\varphi)$ with respect to $\tilde{\sigma}$. Since $\partial \bar{\partial} \lambda(\varphi)=$ $\lambda^{\prime \prime}(\varphi) \partial \varphi \otimes \partial \varphi+\lambda^{\prime}(\varphi) \partial \bar{\partial} \varphi$, noting that the eigenvalues of $\partial \varphi \otimes \bar{\partial} \varphi$ and $\partial \bar{\partial} \varphi$ with respect to $\tilde{\sigma}$ are bounded, we obtain an estimate:

$$
\prod_{i=1}^{n}\left(1+\lambda_{i}\right) \leqq C_{0}\left(\lambda^{\prime}(\varphi)+\lambda^{\prime \prime}(\varphi)\right)^{n},
$$

for some constant $C_{0}$. Hence $\prod_{i=1}^{n}\left(1+\lambda_{i}\right) \leqq C_{1}(\lambda(\varphi))^{4 n}$, since $(\lambda(\varphi))^{2}>\lambda^{\prime}(\varphi)$ and $(\lambda(\varphi))^{4}>\lambda^{\prime \prime}(\varphi)$ outside a compact subset of $X$, where $C_{1}$ is a constant. Therefore,

$$
\begin{aligned}
& \int_{X}|f|_{a_{\lambda}, \sigma_{\lambda}}^{2} d v_{\lambda} \\
& \quad \leqq \int_{X} e^{-\lambda(\varphi)}|f|_{\tilde{a}, \tilde{\sigma}}^{2} d \tilde{v}^{*)} \\
& \quad \leqq \int_{X}\left(e^{-\rho(\varphi)}|f|_{\tilde{a}, \tilde{\sigma}}^{2}\right)\left(e^{-\lambda(\varphi) / 2} \prod_{i=1}^{n}\left(1+\lambda_{i}\right)\right) d \tilde{v}<\infty
\end{aligned}
$$

Thus we obtain $f \in L^{p, q}\left(X, B, a_{\lambda}, \sigma_{\lambda}\right)$.

On the other hand, for any $g \in C_{0}^{p, q}(X, B)$ we have

$$
\begin{aligned}
& \left(\sqrt{-1}\left[e\left(\Theta_{a_{\lambda}}\right), \Lambda_{\sigma_{\lambda}}\right] g, g\right)_{a_{\lambda}, \sigma_{\lambda}} \\
& \quad=\left(\left[L_{\sigma_{\lambda}}, \Lambda_{\sigma_{\lambda}}\right] g, g\right)_{a_{\lambda}, \sigma_{\lambda}} \\
& \quad=(p+q-n)(g, g)_{a_{\lambda}, \sigma_{\lambda}} \geqq\|g\|_{a_{\lambda}, \sigma_{\lambda}}^{2},
\end{aligned}
$$

when $p+q>n$.

Thus, in virtue of Theorem 2.2.3, we can find $u \in L^{p, q}\left(X, B, a_{\lambda}, \sigma_{\lambda}\right)$ such that $\bar{\partial} u=f$.

Remark. Note that the existence of the exhaustion function $\varphi$ is crucial. For example, $\mathbf{C}^{2} \backslash\{0\}$ has a complete Kähler metric but $H^{1}\left(\mathbf{C}^{2} \backslash\{0\}, \Omega_{\mathbf{C}^{2} \backslash\{0\}}^{2}\right)$ does not vanish.

\section{§4. Finite-Dimensionality Theorems}

Since every proper modification of a weakly 1-complete manifold is again weakly 1 -complete, the following theorems would be of some interest.

*) Since $\sigma_{\lambda} \geqq \tilde{\sigma},|v|_{\tilde{\sigma}} \geqq|v|_{\sigma \sigma}$, for any $v \in T_{X}^{*} \otimes \mathbf{C}$. 
Theorem 4.1 (Nakano-Rhai [22]). Let $(X, \varphi)$ be a weakly 1-complete manifold of dimension $n$, and let $(E, h)$ be a hermitian bundle over $X$ whose curvature form is Nakano-positive outside a compact subset of $X$. Then $H^{q}\left(X, \Omega^{n}(E)\right)$ is finite dimensional for $q \geqq 1$.

Theorem 4.2 (Ohsawa [24], [26]). Let $(X, \varphi)$ be a weakly 1-complete manifold of dimension $n$, and let $(B, a)$ be a hermitian line bundle over $X$ whose curvature form is positive outside a compact subset of $X$. Then $H^{q}\left(X, \Omega^{p}(B)\right)$ is finite dimensional when $p+q>n$.

In fact, they are relevant generalizations of Grauert's finiteness theorem. We shall only prove Theorem 4.1 , the proof of Theorem 4.2 being similar in the spirit.

Proof of Theorem 4.1. Fix $c \in \mathbf{R}$ such that $X_{c} \supset K$, and let $K_{1}$ be a compact subset of $X_{c}$ containing $K$ in its interior. We put $h_{c}=h(c-\varphi)$ and fix a hermitian metric $\sigma_{c}$ on $X_{c}$ such that $\sigma_{c}=\Theta_{\text {det } h}+\partial \bar{\partial}(-\log (c-\varphi))$ on $X_{c} \backslash K_{1}$. Replacing $\varphi$ by $c+(\varphi-c) \varepsilon, 0<\varepsilon \ll 1$, if necessary, we may assume that $X_{c-1} \supset K_{1}$. We have as in Section 2 the following estimate:

$$
\|\bar{\partial} f\|_{c}^{2}+\left\|\bar{\partial}^{*} f\right\|_{c}^{2} \geqq \gamma_{0}\|f\|_{c}^{2}, \quad \text { for } \quad f \in C_{0}^{n, q}\left(X_{c} \backslash K_{1}, E\right), q \geqq 1 .
$$

Here, the norm \|\|$_{c}, \bar{\partial}^{*}$ are with respect to $\left(h_{c}, \sigma_{c}\right)$, and $\gamma_{0}$ denotes the infimum on $X_{c} \backslash K_{1}$ of the eigenvalues of $\left(\sigma_{c}^{-1} \otimes i d_{E}\right) \Theta_{h_{c}}$. By Proposition 1.4.4 it is clear that $\gamma_{0}>0$. Applying (7) to $\rho f$, where $f \in C_{0}^{n, q}\left(X_{c}, E\right), \rho$ is a $C^{\infty}$ function such that supp $\rho \Subset X_{c}$ and $\rho=0$ on a neighbourhood of $K_{1}$, we have

$$
C_{1}\left\{\|\bar{\partial} f\|_{c}^{2}+\left\|\bar{\partial}^{*} f\right\|_{c}^{2}+\int_{K_{2}}|f|_{c}^{2} d v_{c}\right\} \geqq\|f\|_{c}^{2}, \quad \text { for } \quad f \in C_{0}^{n, q}\left(X_{c}, E\right) .
$$

Here, $C_{1}$ is a constant and $K_{2}$ is a compact subset of $X_{c}$ containing $K_{1}$. The hermitian metric $\sigma_{c}$ is complete, as we can see it from the inequality $\sigma_{c} \geqq$ $(c-\varphi)^{-2} \partial \varphi \otimes \bar{\partial} \varphi$. Hence by the approximation principle we have

$$
C_{1}\left\{\|\bar{\partial} f\|_{c}^{2}+\left\|\bar{\partial}^{*} f\right\|_{c}^{2}+\int_{K_{2}}|f|_{c}^{2} d v_{c}\right\} \geqq\|f\|_{c}^{2}, \quad \text { for } \quad f \in D_{\bar{\partial}}^{n, q} \cap D_{\bar{\partial}^{*}}^{n, q} .
$$

By strong ellipticity of $\bar{\partial} \vartheta_{h}+\vartheta_{h} \bar{\partial}$, we can apply Garding's inequality for the elements of $C_{0}^{p, q}(X, E)$ (cf. [17]). Hence, by a regularization argument we obtain that for any sequence $\left\{f_{k}\right\} \subset L^{n, q}\left(X_{c}, E, h_{c}, \sigma_{c}\right)$ satisfying $\left\|\bar{\partial} f_{k}\right\|_{c} \rightarrow 0$, $\left\|\bar{\partial} * f_{k}\right\|_{c} \rightarrow 0,\left\|f_{k}\right\|_{c}=1$, and for any $d<c, 1$-st order derivatives of $f_{k}$ are bounded on $X_{d}$ in $L^{2}$-sense. Therefore, by Rellich's lemma, we can find subsequence $\Gamma \subset\left\{f_{k}\right\}$ converging strongly on $K_{2}$. Moreover, by (9), $\Gamma$ converges strongly on 
$X_{c}$. Therefore, by Theorem 1.3.3, $R_{\bar{\partial}{ }^{*}, q}$ is closed for $q \geqq 0$ and $H_{c}^{n, q}:=\{\bar{\partial} f=0$, $\left.\bar{\partial}^{*} f=0\right\}$ is finite dimensional for $q \geqq 1$.

Next, let $\left\{\lambda_{k}\right\}_{k \geqq 1}$ be a sequence of $C^{\infty}$ convex increasing functions such that $\lambda_{k}(t)=-\log (c-t)$ for $t<c-\frac{1}{k}, \lambda_{k}^{\prime}(t)<\frac{1}{c-t}, \lambda_{k}^{\prime \prime}(t)<\frac{1}{(c-t)^{2}}$, for $t<c$, and that $\int_{0}^{\infty} \lambda_{k}^{\prime \prime}(t) d t=\infty$. We fix hermitian metrics $\sigma_{k}$ on $X$ such that $\sigma_{k}=\sigma_{c}$ on $K_{1}$ and $\sigma_{k}=\Theta_{\operatorname{det} h}+\partial \bar{\partial} \lambda_{k}(\varphi)$ on $X \backslash K_{1}$. Then, $\sigma_{k}$ are complete metrics on $X$ and we have the following estimates for $f \in C_{0}^{n, q}\left(X \backslash K_{1}, E\right), q \geqq 1$ :

$$
\|\bar{\partial} f\|_{k}^{2}+\left\|\bar{\partial}^{*} f\right\|_{k}^{2} \geqq\left(\sqrt{-1} e\left(\Theta_{h_{k}}\right) \Lambda_{k} f, f\right)_{k},
$$

where we put $h_{k}=h e^{-\lambda_{k}(\varphi)}$, and \|\|$_{k}, \bar{\partial}^{*}$, etc. are with respect to $\left(h_{k}, \sigma_{k}\right)$. Thus, similarly as in the case of $\left(h_{c}, \sigma_{c}\right)$, we have

$$
\|\bar{\partial} f\|_{k}^{2}+\left\|\bar{\partial}^{*} f\right\|_{k}^{2}=\int_{K_{2}}|f|_{k}^{2} d v_{k} \geqq(\gamma f, f)_{k}, \quad \text { for } \quad f \in D_{\bar{\partial}}^{n, q} \cap D_{\bar{\partial}^{*}}^{n, q}, q \geqq 1 .
$$

Here, $\gamma$ is a positive continuous function on $X$. By Proposition 1.4.4, $\gamma$ can be chosen to be independent of the choice of $\left\{\lambda_{k}\right\}$.

Sublemma. For any $f \in L^{n, q}\left(X, E, h_{k}, \sigma_{k}\right), q \geqq 0$, we have $\|f\|_{k} \geqq\left\|\left.f\right|_{X_{c}}\right\|_{c}$.

Proof. Immediate from the inequalities $\sigma_{k} \leqq \sigma_{c}$ and $h_{k} \geqq h_{c}$.

Assertion. There exist an integer $k_{0}$ and a constant $C_{2}$ such that for any $k \geqq k_{0}$ we have

$$
\begin{aligned}
& C_{2}\left\{\|\bar{\partial} f\|_{k}^{2}+\left\|\bar{\partial}^{*} f\right\|_{k}^{2}\right\} \geqq(\gamma f, f)_{k}, \\
& \quad \text { for } f \in D_{\bar{\partial}}^{n, q} \cap D_{\bar{\partial}^{*}}^{n, q}(q \geqq 1) \text { which satisfy }\left.f\right|_{X_{c}} \perp H_{c}^{n, q} .
\end{aligned}
$$

Proof. Assume the contrary. Then we have a sequence $\left\{f_{k}\right\}_{k=1}^{\infty}$ such that $f_{k} \in L^{n, q}\left(X, E, h_{k}, \sigma_{k}\right),\left\|\bar{\partial} f_{k}\right\|_{k} \rightarrow 0,\left\|\bar{\partial}^{*} f_{k}\right\|_{k} \rightarrow 0,\left(\gamma f_{k}, f_{k}\right)_{k}=1$, and that $\left.f_{k}\right|_{X_{c}} \perp H_{c}^{n, q}$. From (11), as before we can choose a subsequence $\mathscr{S} \subset\left\{f_{k}\right\}$ converging strongly on $K_{2}$ to a non-zero form. Choose a subsequence $\left\{f_{k_{i}}\right\} \subset \mathscr{S}$ such that $\left\{\left.f_{k_{i}}\right|_{X_{c}}\right\}$ converges weakly in $L^{n, q}\left(X_{c}, E, h_{c}, \sigma_{c}\right)$ (cf. Sublemma). Let the weak limit be $f$. Then $f \neq 0$ and $f \perp H_{c}^{n, q}$. But, since $\left\|\bar{\partial} f_{k}\right\|_{k} \geqq\left\|\left.\bar{\partial} f_{k}\right|_{X_{c}}\right\|_{c}$, we have $\bar{\partial} f=0$, and moreover $(f, \bar{\partial} u)_{c}=\lim \left(f_{k}, \bar{\partial} u\right)_{k}=\lim \left(\bar{\partial}^{*} f_{k}, u\right)_{k}=0$ for any $u \in C_{0}^{n, q-1}\left(X_{c}, E\right)$, so that $\bar{\partial} * f=0$. It is a contradiction.

Thus, by Theorem 1.3.2, for any $g \in N_{\frac{n}{\partial}, q} \cap L^{n, q}\left(X, E, h_{k}, \sigma_{k}\right) \quad(q \geqq 1)$, satisfying $\left.g\right|_{X_{c}} \perp H_{c}^{n, q}$ and $\int_{X} \gamma^{-1}\langle g, g\rangle_{k} d v_{k}<\infty$ for some $k \geqq k_{0}$, we can find $u \in L^{n, q-1}\left(X, E, h_{k}, \sigma_{k}\right)$ such that $\bar{\partial} u=g$. Since the growth of $\lambda_{k}$ outside $(-\infty, c)$ can be chosen to be arbitrarily rapid, we conclude that for any $g \in L_{l o c}^{n, q}(X, E)$, 
$q \geqq 1$, satisfying $\left.g\right|_{X_{c}} \perp H_{c}^{n, q}$ and $\bar{\partial} g=0$, we can find $u \in L_{l o c}^{n, q-1}(X, E)$ satisfying $\bar{\partial} u=g$.

Thus we have proved that the composite of restriction and harmonic projection $H^{q}\left(X, \Omega^{n}(E)\right) \rightarrow H_{c}^{n, q}$ is injective for $q \geqq 1$. Since $H_{c}^{n, q}$ is finite-dimensional as we have proved earlier, $H^{q}\left(X, \Omega^{n}(E)\right)$ is also finite-dimensional.

\section{\$5. Other Results}

Originally, Theorem 4.1 was proved via the following two theorems which are interesting themselves.

Theorem 5.1. Let $(X, \varphi)$ be a weakly 1-complete manifold of dimension $n$, and let $(E, h)$ be a hermitian vector bundle over $X$ whose curvature form is Nakano-positive outside a compact subset $K \subset X$. Then, for any $X_{c}$ which contains $K$, the natural restriction maps

$$
\rho_{c}: H^{q}\left(X, \Omega^{n}(E)\right) \longrightarrow H^{q}\left(X_{c}, \Omega^{n}(E)\right)
$$

have dense images for $q \geqq 0$. Here the topology of $H^{q}\left(X_{c}, \Omega^{n}(E)\right)$ is induced from $L_{l o c}^{n, q}\left(X_{c}, E\right)$.

Sketch of Proof. Let $X_{c} \supset X_{c^{\prime}} \supset K$ and let $u \in L^{n, q}\left(X_{c^{\prime}}, E, h_{c^{\prime}}, \sigma_{c^{\prime}}\right)$ with $u \perp\left\{\left.f\right|_{X_{c^{\prime}}} \mid f \in L_{l o c}^{n, q}(X, E), \bar{\partial} f=0\right\}$. Then, if we extend $u$ outside $X_{c^{\prime}}$ by 0 and define $u_{k} \in L^{n, q}\left(X, E, h_{k}, \sigma_{k}\right)$ by " $\left(u,\left.v^{\prime}\right|_{X_{c^{\prime}}}\right)_{c^{\prime}}=\left(u_{k}, v^{\prime}\right)_{k}$ for any $v^{\prime} \in L^{n, q}(X$, $\left.E, h_{k}, \sigma_{k}\right)$ ', then we have $u_{k}=\bar{\partial}^{*} v_{k}$ for some $V_{k}$ with $\left\|v_{k}\right\|_{k} \leqq\left\|u_{k}\right\|_{k} \leqq\|u\|_{c^{\prime}}$. Let the weak limit of a subsequence of $\left\{\left.v_{k}\right|_{X_{c}{ }^{\prime}}\right\}$ be $v$. Then we have $u=\bar{\partial}^{*} v$, so that $u \perp N^{n, q}$.

Since $H^{q}\left(X_{c}, \Omega^{n}(E)\right)$ are finite-dimensional for $q \geqq 1$, we have thus proved that $\rho_{c}$ are surjective for $q \geqq 1$. When $q=0$, Theorem 5.1 amounts to a generalization of the classical theorem of Runge. By the same argument we can prove that the map $H^{q}\left(X, \Omega^{n}(E)\right) \rightarrow H_{c}^{n, q}$ have dense image for $q \geqq 0$. Hence, for $q \geqq 1, H^{q}\left(X, \Omega^{n}(E)\right)$ is isomorphic to $H_{c}^{n, q}$. Thus we obtain

Theorem 5.2. Let the situation be as above. Then, the natural restriction maps $\rho_{c}$ are isomorphisms for $q \geqq 1$.

Similarly we have

Theorem 5.3 (cf. [24]). Let the situation be as above, and let the rank of $E$ be 1 . Then the maps $\rho_{c}: H^{q}\left(X, \Omega^{p}(E)\right) \rightarrow H^{q}\left(X_{c}, \Omega^{p}(E)\right)$ have dense images for $p+q \geqq n$, and are isomorphisms for $p+q>n$. 


\section{§6. Applications}

Let $M$ be a complex manifold and let $D \subset M$ be a relatively compact domain with $C^{2}$-smooth boundary $\partial D$. Let $\psi$ be a function of class $C^{2}$ on $M$ such that $D=\{x \in M \mid \psi(x)<0\}$ and that $d \psi \neq 0$ everywhere on $\partial D$. Then $\partial D$ is said to be pseudoconvex (strongly pseudoconvex) if $\partial \bar{\partial} \psi(\xi, \bar{\xi}) \geqq 0(>0)$ for any $\zeta \in T_{M, x}^{1,0} \backslash\{0\}, x \in \partial D$, satisfying $\partial \psi(\xi)=0$

Theorem 6.1 (Grauert [10]). If $D$ is a domain with strongly pseudoconvex boundary, then $D$ is holomorphically convex.

As a corollary we have

Theorem 6.2. If $X$ is a strongly pseudoconvex manifold, then $X$ is holomorphically convex.

Proof. Let $\Phi$ be an exhaustion function which is strictly plurisubharmonic outside a compact subset of $X$. Then we can choose by Sard's theorem an increasing sequence of real numbers $\left\{c_{i}\right\}_{i=1}^{\infty}$ such that $c_{i}$ are non-critical values of $\Phi$ and that $\left\{\Phi=c_{i}\right\}$ are strongly pseudoconvex. By Theorem 6.1, $D_{i}:=\left\{\Phi<c_{i}\right\}$ are holomorphically convex. Hence, for any $i<j,\left(D_{i}, D_{j}\right)$ is a Runge-pair, whence follows immediately the holomorph-convexity of $X=\cup_{i=1} D_{i}$.

We shall give a direct proof of Theorem 6.2 as an application of Theorem 4.1. Since every domain with strongly pseudoconvex boundary is a strongly pseudoconvex manifold, (cf. [13]), Theorem 6.1 is then a special case of Theorem 6.2, and we need no approximation theorem of Runge type.

Quadratic transformation: Let $X$ be a complex manifold of dimension $n$, and let $x \in X$ be any point. Then, there exists a unique complex manifold $Q_{x} X$ which have the following properties (cf. [32]).

i) There is a proper holomorphic map $\pi_{x}: Q_{x} X \rightarrow X$ which is one to one outside $\pi_{x}^{-1}(x)$.

ii) $\pi_{x}^{-1}(x) \cong \mathbf{P}^{n-1}$.

$Q_{x} X$ is called the quadratic transform of $X$ centered at $x$. Let $\left[\pi_{x}^{-1}(x)\right]$ be the line bundle associated to the divisor $\pi_{x}^{-1}(x)$. Then, $\left.\left[\pi_{x}^{-1}(x)\right]\right|_{\pi_{x}^{-1}(x)} \cong H_{n-1}^{-1}$, where $H_{n-1}$ denotes the hyperplane bundle over $\mathbf{P}^{n-1}$. For distinct points $x, y \in X$, we have $Q_{\pi_{v}^{-1}(x)}\left(Q_{y} X\right) \cong Q_{\pi_{x}^{-1}(y)}\left(Q_{x} X\right)$. Thus we can put $Q_{\{x, y\}} X:=$ 
$Q_{\pi_{x}^{-1}(y)}\left(Q_{x} X\right)$. Similarly we define $Q_{I} X$ and $\pi_{I}: Q_{I} X \rightarrow X$ for any discrete set of points $I \subset X$.

Lemma 6.3.

$$
\left.\left(\wedge^{n} T_{Q_{x} X}^{1,0}\right)\right|_{\pi_{x}^{-1}(x)} \cong H_{n-1}^{n-1}
$$

Proof. See [32].

Proof of Theorem 6.2. Let $(X, \varphi)$ be a non-compact strongly pseudoconvex manifold of dimension $n$ with a $C^{\infty}$ plurisubharmonic exhaustion function. Let $I=\left\{x_{i}\right\}_{i=1}^{\infty} \subset X$ be any discrete sequence. Then, by Lemma 6.3, the line bundle $\left.\left(\wedge^{n} T_{Q_{I} X}^{1,0}\right) \otimes \pi_{I}^{-1}(I)\right]^{*}$ admits a hermitian metric $a$ whose curvature form $\Theta$ satisfies $\Theta(v, \bar{v})>0$ for any $v \in T_{X}^{1,0}$ which are tangent to $\pi_{I}^{-1}(I)$. Hence we can find a $C^{\infty}$ convex increasing function $\lambda: \mathbf{R} \rightarrow \mathbf{R}$ such that the curvature form of $a \exp \left(-\lambda\left(\pi_{I}^{*} \varphi\right)\right)$ is positive outside a compact subset of $Q_{I} X$. Thus, $H^{1}\left(Q_{I} X\right.$, $\left.\mathcal{O}\left(\left[\pi_{I}^{-1}(I)\right]^{*}\right)\right)=H^{1}\left(Q_{I} X, \Omega^{n}\left(\wedge^{n} T_{X}^{1,0} \otimes\left[\pi_{I}^{-1}(I)\right]^{*}\right)\right)$ is finite dimensional. For the structure sheaf $\mathcal{O}_{Q_{I} X}$ we have the following exact sequence:

$$
\begin{aligned}
& \Gamma\left(X, \mathcal{O}_{X}\right) \\
& \quad \|\left(Q_{I} X, \mathcal{O}_{Q_{I} X}\right) \longrightarrow \Gamma(I, \mathbf{C}) \longrightarrow H^{1}\left(Q_{I} X, \mathcal{O}\left(\left[\pi^{-1}(I)\right]^{*}\right)\right) .
\end{aligned}
$$

Since $H^{1}\left(Q_{I} X,\left[\pi^{-1}(I)\right]^{*}\right)$ is finite dimensional, by the above exact sequence we can find $f \in \Gamma\left(X, \mathcal{O}_{X}\right)$ such that $\left|f\left(x_{i}\right)\right| \rightarrow \infty$ when $i \rightarrow \infty$.

Similarly as above we obtain

Theorem 6.4. Let $X$ be a weakly 1-complete manifold and let $S \subset X$ be a divisor. Assume that $\wedge^{n} T_{X}^{1,0} \otimes[S]^{-1}$ has a hermitian metric whose curvature form is positive. Then, every holomorphic function on $S$ is holomorphically extendable to $X$.

Proof. Immediate from the following exact sequence:

$$
\begin{gathered}
\Gamma\left(X, \mathcal{O}_{X}\right) \longrightarrow \Gamma\left(S, \mathcal{O}_{S}\right) \longrightarrow H^{1}\left(X, \mathscr{O}_{\|}\left([S]^{-1}\right)\right) \\
H^{1}\left(X, \|_{\Omega^{n}}\left(\|^{n} T_{X}^{1,0} \otimes[S]^{-1}\right)\right) \\
0,
\end{gathered}
$$

(cf. Theorem 3.6).

It follows from Theorem 6.4 that some divisors can be contracted analytically to lower dimensional analytic subsets. In particular, we have

Theorem 6.5. Let $D \rightarrow M$ be a holomorphic $\mathbf{P}^{n}$-bundle over a connected complex manifold $M$ and let $D \hookrightarrow X$ be an embedding of $D$ as a divisor. If the 
degree of the bundle $\left.[D]\right|_{\pi^{-1}(p)}$ is -1 for some (hence for any) $p \in M$, then there exists a complex manifold $Y$ and a proper holomorphic map $\tilde{\pi}: X \rightarrow Y$ such that $\tilde{\pi}$ is one to one outside D.

Proof. See Nakano [19].

Remark. Theorem 6.5 has been considerably generalized by Fujiki [9] and Bingener [7] (see also Ancona-Tomassini [33]).

In the same spirit as above, we have shown in [25],

Theorem 6.6. Let $X$ be a weakly 1-complete manifold of dimension two. Assume that $\stackrel{2}{\wedge} T_{X}^{1}, 0$ has a hermitian metric whose curvature form is positive. Then $X$ is holomorphically convex.

Combining Theorem 6.6 with Theorem 3.6 , we can prove

Theorem 6.7 (cf. Theorem 2.1 and Theorem 2.3 in [25]). Let $Y$ be either a hypersurface in $\mathbf{P}^{n}$ of degree less than 4 , or a complete intersection of type $(2,2)$ in $\mathbf{P}^{n}$, and let $X$ be an unramified domain over $Y$. Then, $X$ is holomorphically convex if and only if $X$ is weakly 1-complete.

As for the projective embeddability of weakly 1-complete manifolds, we have

Theorem 6.8. Let $(X, \varphi)$ be a weakly 1-complete manifold and let $(B, a)$ be a positive line bundle over $X$. Then, for any $c \in \mathbf{R}$, there exist an integer $m$ and a holomorphic embedding of $X_{c}$ into $\mathbf{P}^{N}$, where $N$ depends on $c$.

Under the situation of Theorem 6.8 , whether $X$ is embeddable into some $\mathbf{P}^{N}$ or no is an open problem. By now the following is the unique result in this direction.

Theorem 6.9 (Takegoshi [30]). Let $\operatorname{dim} X=2 . \quad$ Assume that $X$ contains only finitely many exceptional curves, then $X$ is holomorphically embeddable into $\mathbf{P}^{5}$.

\section{§7. Variations of Vanishing Theorems}

Let $(X, \varphi)$ be a weakly 1-complete manifold of dimension $n$, let $(B, a)$ be a hermitian line bundle over $X$, and let $\Theta$ be the curvature form of $a$. There are several variations of Theorem 3.6 and Theorem 3.7.

Theorem 7.1 (Abdelkader [1], [2], Takegoshi-Ohsawa [31], Skoda [27]). 
Assume that $X$ has a Kähler metric. If $\Theta$ is positive semi-definite and rank $\Theta$ $\geqq n-k+1$ everywhere, then

$$
H^{q}\left(X, \Omega^{p}(B)\right)=0 \quad \text { when } \quad p+q \geqq n+k .
$$

Theorem 7.2 (Ohsawa [26]). If $\Theta$ has at least $n-k+1$ positive eigenvalues, then, for any holomorphic vector bundle $E \rightarrow X$ and for any $c \in \mathbf{R}$, there exists an integer $m_{0}$ such that

$$
H^{q}\left(X_{c}, \mathcal{O}\left(E \otimes B^{m}\right)\right)=0, \quad \text { for } \quad q \geqq k \text { and } m \geqq m_{0} .
$$

Theorem 7.3 (Ohsawa [26]). Assume that $X$ is Kählerian and that rank $\partial \bar{\partial}\left(e^{\varphi}\right) \leqq r$. If $\Theta$ is negative semi-definite and has rank at least $n-k+1$, then

$$
H^{q}\left(X, \Omega^{p}(B)\right)=0, \quad \text { for } \quad p+q \leqq n-k-r .
$$

Takegoshi applied Aronszajn's unique continuation theorem [6] for $\bar{\partial} \vartheta_{h}+$ $\vartheta_{h} \bar{\partial}$ to obtain

Theorem7.4 (cf. [29]). Assume that $X$ is connected and Kählerian, and that $\Theta \geqq 0$. If $\Theta>0$ outside a compact subset of $X$, then

$$
H^{q}\left(X, \Omega^{n}(B)\right)=0, \quad \text { for } \quad q \geqq 1 .
$$

Finite-dimensionality theorems analoguous to Theorem 4.2 are also valid. See Ohsawa [26] and Abdelkader [3].

\section{References}

[1] Abdelkader, O., Annulation de la cohomolgie d'une variete kählerienne faiblement 1-complete a valeur dans un fibre vectoriel holomorphe semi-positif, C. R. Acad. Sci. Paris, 290 (1980), 75-78.

[2] - Un theoreme d'approximation pour les formes a valeurs dan un fibre semipositif. C. R. Acad. Sci. Paris, 293 (1981), 513-515.

[3] - Generalisation d'un theorem de finitude, C. R. Acad. Sci. Paris, 293 (1981), 629-632.

[4] Andreotti, A. and Vesentini, E., Sopra un teorema di Kodaira, Ann. Sci. Norm. Sup. Pisa, (3), 15 (1961), 283-309.

[ 5 ] — Carleman estimates for the Laplace-Beltrami equation on complex manifolds, Publ. Math. IHES, 25 (1965), 81-130.

[6] Aronszajn, N., A unique continuation theorem for solutions of elliptic partial differential equations or inequalities of second order, J. Math. Pure. Appl., 36 (1957), 235-249.

[7] Bingener, J., On the existence of analytic contractions, Inventiones math. 64 (1981), 25-67.

[ 8 ] Fujiki, A. and Nakano, S., Supplement to "On the inverse of monoidal transformation" Publ. RIMS, Kyoto Univ., 7 (1971-72). 637-644.

[9] Fujiki, A., On the blowing down of analytic spaces, Publ. RIMS, Kyoto Univ., 10 
(1975), 473-507.

[10] Grauert, H., On Levi's Problem and the imbedding of real-analytic manifolds, Ann. of Math. 68, (1958), 460-472.

[11] Grauert, R., and Riemenschneider, O., Kählersche Mannigfaltigkeiten mit hyper-qkonvexem Rand, Problems in Analysis, Princeton Univ. Press, Princeton, N. J., (1970), 61-70.

[12] Griffiths, P. H., Hermitian differential geometry, Chern classes, and positive vector bundles, Global analysis, Univ. of Tokyo Press and Princeton Univ. Press., (1969), 185-251.

[13] Gunning, R. C., and Rossi, H., Analytic functions of several complex variables, Englewood Cliffs, N. J., Prentice Hall Inc., 1965.

[14] Hörmander, L., $L^{2}$ estimates and existence theorems for the $\bar{\partial}$ operator, Acta Math., 113 (1965), 89-152.

[15] Kazama, A., Approximation theorem and application to Nakano's vanishing theorems for weakly 1-complete manifolds, Men. Fac. Sci. Kyushu Univ., 27 (1973), 221-240.

[16] Kodaira, K., On a differntial geometric method in the theory of analytic stacks, Pro. Nat. Acad. Sci. U. S. A., 39 (1953), 1269-1273.

[17] Morrey, C. B., Multiple integrals in the calculus of variations, Springer, 1966.

[18] Nakano, S., On complex analytic vector bundles, J. M. S. Jap., 7 (1955), 1-12.

[19] - On the inverse of monoidal transformation, Publ. PIMS, Kyoto Univ. $6(1970 / 71), 483-502$.

[20] - Vanishing theorems for weakly 1-complete manifolds, Number theory, Algebraic geometry, and Commutative algebra, Kinokuniya, Tokyo, 1973, 169-179.

[21] —, Vanishing theorems for weakly 1-complete manifolds II, Publ. RIMS, Kyoto Univ., 10 (1974), 101-110.

[22] Nakano, S., and Rhai, T.S., Vector bundle version of Ohsawa's finiteness theorems, Math. Japonica, 24 (1980), 657-664.

[23] Ohsawa, T., Finiteness theorems on weakly 1-complete manifolds, Publ. RIMS, Kyoto Univ., 15 (1979), 853-870.

[24] - On $H^{p, q}(X, B)$ of weakly 1-complete manifolds, Publ, RIMS, Kyoto Univ., 17 (1981), 113-126.

[25] Weakly 1-complete manifold and Levi problem, Publ. RIMS, Kyoto Univ., 17 (1981), 153-164.

[26] - Isomorphism theorems for cohomology groups of weakly 1-complete manifolds, Publ. RIMS, Kyoto Univ., 18 (1982) 191-232.

[27] Skoda, H., Remarques a propos des theoremes d'annulation pour les fibres semipositifs, Lecture Notes in Mathematics, 822 (1980), 252-257.

[28] Suzuki, O., Simple proofs of Nakano's vanishing theorems for weakly 1-complete manifolds, Publ. RIMS, Kyoto Univ., 17 (1975), 201-211.

[29] Takegoshi, K., A generalization of vanishing theorems for weakly 1-complete manifolds, Publ. RIMS, Kyoto Univ., 17 (1981), 311-330.

[30] - On weakly 1-complete surfaces without non-constant holomorphic functions, Publ. RIMS, Kyoto Univ, 18 (1982), to appear.

[31] Takegoshi, K., and Ohsawa, T., A vanishing theorem for $H^{p}\left(X, \Omega^{q}(B)\right)$ on weakly l-complete manifolds, Publ. RIMS, Kyoto Univ., 17 (1981), 723-733.

[32] Wells, R. O., Differential analysis on complex manifolds, Prentice Hall, Englewood Cliffs, N. J., 1973.

[3.3] Ancona, V. and Tomassini, G., Modification analytiques. Lecturc Notes in Mathematics, 943 (198?). 
